\title{
HACIA UNA VISIÓN DE ALTERIDAD EN FAMILIAS CON POBREZA ECONÓMICA
}

\section{TOWARDS A VISION OF ALTERITY IN FAMILIES WITH ECONOMIC POVERTY}

\author{
Freddy González Silva*
}

RESUMEN

En esta investigación se emplea el constructo "alteridad" entendido desde las interacciones humanas presentes dentro del núcleo familiar. El abordaje fue en un contexto venezolano caracterizado por la pobreza económica. Así pues, con base en una encuesta a 102 familias se obtuvo indicadores de alteridad tales como el matricentrismo, la alteración entre hermanos, la tensión y el encuentro familiar. A partir del análisis de los resultados se formularon nuevas orientaciones para la intervención psicológica, social y educativa. Finalmente se pretendió dar cuenta de las implicaciones de una nueva perspectiva de estudio acerca de las familias.

PALABRAS CLAVE: VENEZUELA * FAMILIA * ALTERIDAD * RELACIONES FAMILIARES * POBREZA

\section{ABSTRACT}

In this investigation is used the construct "alterity" (understood as the subjective experience in the human being in relation to another). The term, which comes from "alter ego", is used to deepen in the present human interactions within the family nucleus. The context was a Venezuelan milieu characterized by the economic poverty. Therefore, based on a survey of 102 families, the alterity indicators obtained were such as the mother-centeredness (understood like the cultural tradition of the home centered by the maternal figure like essential figure of authority), conflicts between brothers, family tensions and the family encounters. From the analysis of the results new directions for the psychological, social and educational interventions were formulated. Finally the implications of a new perspective for family was brought to account.

KEY WORDS: VENEZUELA * FAMILY * ALTERITY * FAMILIAR RELATIONS * POVERTY

Facultad de Ingeniería, Universidad Central de Venezuela- Núcleo Cagua, adscrito al
Departamento de Enseñanzas Generales. gastongonzalez7@yahoo.com 


\section{INTRODUCCIÓN}

La familia, como cualquier otra institución de la sociedad, va cambiando y eso amerita su constante estudio y revisión. Ariza y De Oliveira (2001) plantean que la dinámica familiar se transforma en la medida que cambian los patrones culturales. De esta manera las transformaciones macroestructurales generan cambios en la forma en que se perciben y autoperciben los núcleos familiares. De allí que es vital reconocer cómo es la visión que tienen los propios miembros de la familia acerca de sus integrantes, es decir, como se plantea la alteridad familiar.

La postura acerca del otro es una dimensión comúnmente estudiada para tratar temas de exclusión o de intolerancia (Rodríguez, 2003; Arruda, 1998; González, 2005). Pero una visión amplia de esta categoría se halla en la manera ética de convivir con los demás. Caride (2006) expresa que alteridad es la relación del ser con el otro, "mi relación con el otro" igual pero distinto, que incluye la capacidad ética de reconocerlo y responsabilizarse de quién es, de lo que hace o desea.

Así pues, al tratar la alteridad se hace referencia a otro el cual se reconoce desde la diferencia. Krotz (1994) señala que no es sinónimo de una simple y sencilla diferenciación. En efecto, no se trata de la constatación de que todo ser humano es un individuo único $y$ que siempre se pueden encontrar algunas diferencias en comparación con cualquier otro ser humano (constatación de diferencias pasajeras o variantes de naturaleza física, psíquica y social). Alteridad significa un tipo particular de diferenciación. Tiene que ver con la experiencia de lo extraño. Así pues, Vila (2004) señala que la alteridad emerge desde el encuentro entre lo propio y lo otro desconocido que se hace presente frente a nosotros.

Tratar la alteridad desde la familia conlleva a preguntarse acerca de la forma en que se perciben unos a otros dentro de esta agrupación. En la familia todos se conocen constantemente. Esta acción de descubrirse es afectiva y cognoscitiva, según Colmenares (2004). Evidentemente en el hogar se van captando características escondidas o ignoradas de cada uno de los miembros a medida que transcurre el tiempo. Importa reconocer que son algunos integrantes quienes perciben más la presencia del otro.

De todas formas lo cierto es que se da un autoconocimiento y reconocimiento entre hijos, padres, hermanos, etc. En términos de Levinas (1993) no es una relación idílica y armoniosa de comunión, ni una empatía; si no se le reconoce como semejante al yo y al mismo tiempo exterior.

Visto de esta forma el proceso va asociado con el establecimiento de diferencias entre sus identidades. Finalmente cada yo integra esta comprensión a su experiencia individual y termina formando parte de su persona. De allí que se expresará en la forma en que cada uno interactúa con respecto a los otros. Este asunto en el nivel familiar es a lo que se le puede llamar condición de alteridad.

Para el caso en cuestión este tema no ha sido abordado exactamente desde esta perspectiva pero si se encontraron diferentes estudios acerca de la importancia de tratarlo. Es fundamental profundizar en el conocimiento de relaciones bidireccionalmente amistosas dentro del hogar ya que, como señala Laursen, (2005) esto hace que el futuro de los integrantes tienda a la socialización.

Al ser una temática no común se presentan escasos instrumentos para evaluarla. Arraigada (2005) señala la necesidad de nuevos medios para medir la calidad de vida familiar que se basen en la interacción entre sus miembros y permitan evaluar realmente la dinámica familiar. Igualmente insta a que se evalúe la violencia intrafamiliar.

Importa aclarar que la investigación acerca de la familia tiene muchas facetas aún por develar. Miguel y Vargas (2001) plantean que hablar de familia no constituye ubicarse en el paradigma tradicional de su concepción sino por el contrario obtener insumos acerca de la representación social de la misma. Es así como tratar la familia pasa por un conjunto de percepciones obtenidas gracias a la alteridad existente no solo entre sino hacia los miembros del grupo.

En efecto, repensar la familia desde las interpretaciones que sus miembros hacen de 
sí es de alguna manera entender la familia en clave de alteridad. Amara (1993) explicita que la familia es un grupo que se estructura mediante una historia de relaciones verbales o no verbales que contienen significados. En todo caso puede señalarse que esa manera de percibirse entre un grupo de convivencia hace que se generen comunicaciones particulares entre esa familia.

De cualquier manera es prioritario abordar la temática de la alteridad acerca de la familia. Galán y Pinzón (2002) evidencian como para los estudios familiares la observación de sus interacciones son un plano ineludible.

Venezuela no escapa a la insuficiente investigación sobre los núcleos familiares. Algunos trabajos versan sobre la familia en la clase media y sus realidades (De Viana, 1997). No obstante, estas dimensiones no son totalmente únicas sino que colindan histórica y culturalmente con el resto de familias pertenecientes a distintos estratos sociales.

En este sentido se comprende como los estudios actuales se hayan revestidos de críticas debido a la complejidad del tema. Moreno (2004a) plantea que el discurso que ha rodeado la investigación acerca de la familia venezolana siempre la ha definido como desestructurada y pobre. Así pues, se trata de conocimientos adquiridos por una elite de investigadores poco cercanos a la realidad popular. Por esto en algunos casos resulta ser una investigación sesgada. De allí que hace falta un estudio encarnado en la realidad del país, que no descuide el acercamiento cuidadoso del propio subjetivismo producido por esta temática en el investigador. Dentro de este marco se ha tomado como prioritaria una investigación acerca de las familias pobres.

Por lo demás cabe preguntarse: ¿en qué medida se presenta la alteridad en la familia venezolana ubicada en sectores de pobreza económica? De esta manera se podría esclarecer situaciones $y$ formular lineamientos para la acción.

\section{METODOLOGÍA}

La investigación fue de carácter descriptiva. Se realizó siguiendo un diseño: de campo, transeccional contemporáneo y con fuentes vivas.
PARTICIPANTES. Los sujetos participantes fueron 102 familias que viven en la pobreza, carentes principalmente de recursos económicos necesarios para la subsistencia. Se hallan ubicadas en el municipio Sucre del Estado Aragua de Venezuela.

Importa por muchas razones para la interpretación posterior de los resultados, tomar en cuenta como es el medio. En efecto el tema en estudio sobre la alteridad entre el núcleo familiar se encuentra relacionado con su interacción familia-ambiente, cómo viven, qué calidad de vida poseen. De allí se podrá comprender mejor las bases psicológicas, sociales, económicas, entre otras; dado que rodean ese tipo de procesos entre los miembros del grupo familiar. Por tanto se conocerá el exosistema en donde se desenvuelve el sistema familiar.

Por lo demás cabe aclarar que se trata de familias numerosas $(52 \%$ constituidas por $6 y$ hasta 10 integrantes) con igual distribución de miembros por género. En formación educativa se percibe un $4 \%$ de ingreso a la universidad y un $14 \%$ de secundaria completa. Aun se presenta el analfabetismo (un 3\%). En cuanto a la edad se halla una natalidad en descenso y un 39\% de hijos en edades de 7 a 18 años. La mitad de los integrantes aptos para trabajar son desempleados.

Un grupo de familias posee distintas conformaciones y solo el $42 \%$ es de carácter tradicional. Así pues, el resto lo conforman familias monoparentales (23\%) y grupos familiares (35\%). Disponen de escaso espacio físico y solo un $60 \%$ posee casas. Un $17 \%$ de estas familias posee miembros discapacitados y la mayoría están afectadas por problemas respiratorios, digestivos y dérmicos (88\%).

INSTRUMENTOS. Para este estudio la técnica utilizada fue la encuesta. De allí que el instrumento estuvo constituido por un cuestionario compuesto de 29 ítems (24 cerrados y 5 abiertos). Dicho instrumento fue aplicado en su mayoría por personas que viven dentro de la comunidad $(80 \%)$ luego de pasar por un entrenamiento intensivo. También se empleó para la triangulación, el diario del investigador basado en los datos que se recogen gracias a la observación durante la aplicación de la encuesta. 
TIPO DE ANÁLISIS UTILIZADO. El tipo de análisis utilizado tomó en cuenta dos aspectos: a) las frecuencias de los porcentajes de respuestas entre el criterio de análisis y el evento analizado, obtenidos de la matriz y de los cuestionarios y b) el análisis verbal dado por la reinterpretación de los contenidos en función del criterio, los cuales se obtuvieron de todos los instrumentos. Desde esta perspectiva se llevó a cabo un proceso de triangulación. Cisterna (2005) señala que el proceso de triangulación consiste en la acción de reunión y cruce dialéctico de toda la información pertinente al objeto de estudio surgida en una investigación por medio de los instrumentos correspondientes y que en esencia constituye el corpus de resultados de la investigación.

\section{ANÁLISIS DE LOS RESULTADOS}

El presente estudio permite agrupar los datos en cuatro dimensiones de alteridad que se describen a continuación:

\section{ALTERIDAD CON ÉNFASIS EN EL MATRICENTRISMO}

En el estudio se observa como la madre (o cualquier mujer del grupo que reciba esta denominación) dentro del hogar ocupa un eje desde donde se reflexiona la otredad. La progenitora es quien percibe los cambios (60\%). De igual manera es la madre quien genera el mayor nivel de solidaridad (33\%). También es desde ella quien expresa mayor apertura (32\%). Así mismo es la encargada de manejar la información del hogar en un gran número de situaciones y la que mejor puede explicar el ego de cada miembro ( $\left.\mathrm{R}^{1} 28\right)$. Desde esta perspectiva esta mujer es la primera percibida en los hogares $y$ es ella la que asume internamente la presencia de los otros de manera intensa.

1 La R significará reporte verbal obtenido de los diarios de investigación.

\section{DESVINCULACIÓN ENTRE LA PAREJA}

Así mismo se encontró que las relaciones se dan entre cualesquiera de los miembros con mayor porcentaje que a nivel de la pareja (7\%). La pareja no constituye una proxemia que implique una hermenéutica de alteridad. Aunado a esto se presenta una situación de celos (17\%).

\section{ALTERIDAD ALTERADA FUNDAMENTALMENTE ENTRE HERMANOS}

La alteridad real es el otro distinto que como señala Theodosíadis (1996) traspasa el horizonte ontológico de la totalidad humana. Visto de esta forma surge una relación que se matiza de acuerdo con una evolución personal. En esta perspectiva se encontró que los miembros familiares generadores de discordias entre sí eran los hermanos (41\%), los menores de la familia.

Así se ha verificado como los hermanos generan alteridad desde la diferencia (28\%) la cual es asumida como conflicto. Sin embargo, no se trata de una situación que suscita exclusión o autoexclusión (R27).

También es cierto que la mayoría de los hermanos se encuentran entre los siete y dieciocho años. Así se confirman los planteamientos de Motrico et ál. (2001) quienes afirman una consecuente disfunción en las interacciones familiares cuando los hijos llegan a la adolescencia. En esta etapa parece haber una alteridad familiar muy distinta.

$\mathrm{El}$ análisis procedente conduce a estar de acuerdo con Valadez et ál. (2005) quienes expresan que la familia está llamada a desempeñar una nueva función de roles porque es un espacio dinámico que no puede permanecer estático. Se antepone sobre todo el desarrollo psicológico de cada uno de sus miembros. De allí que el entramado relacional y la manera de percibirse unos a otros sea distinto a los de cualquier otro miembro en la familia. 


\section{TENSIÓN FAMILIAR}

Las situaciones de desequilibrio familiar surgen en un momento de alteridad distorsionada. Stein (2004) señala que la alteridad es el otro frente al otro simple y sin distinción cualitativa. Por consiguiente este interactuar entre ambos se vuelve empatía.

Ahora bien, en el núcleo de las familias estudiadas se presentan con frecuencia peleas, gritos, insultos y golpes (72\%). De este modo se produce una tensión familiar prolongada $y$ delicada. Amara (1993) señala que los conflictos en una familia forman la base para una especie de memoria familiar. Sin duda los miembros de una familia mantienen vivos recuerdos de sus relaciones en sus grupos familiares de origen. Igualmente esta historia influirá en sus futuras relaciones.

Debe señalarse que muchas de las familias estudiadas crecen con miembros drogadictos, ausencias de padre o madre o de ambos, exclusión escolar, deserción escolar, trabajo infantil, abuso sexual o psicológico, desempleo y embarazo precoz (77\%). Los ambientes donde se genera la relación humana presentan desorden, hacinamiento, promiscuidad e insalubridad (70\%). Habida cuenta de esto, no se deben olvidar los planteamientos de Kliksberg (2005) quien acota que la realidad económica es fundamental y tensa en demasía, las relaciones familiares.

Para la mayoría de los miembros los misterios (tabúes, secretos) presentes en sus familias van más allá de situaciones sexuales y de muerte (85\%) lo cual genera lagunas en el estudio que aun faltan por develar. Solo un pequeño número de miembros percibe la tensión en su realidad familiar (23\%) y considera en segundo plano que es un asunto a resolver (R25, 21\%).

En este mismo orden de ideas se reconoce como la alteridad se vuelve ecpática en lugar de empática. En términos de González (2004) la ecpatía consiste en la exclusión de motivaciones, pensamientos, sentimientos y actitudes inducidas por el otro. Por consiguiente la alteridad se vuelve tensión.

Hay sin embargo, otras situaciones que no representan mayores porcentajes pero que dan señales de alerta acerca de estas familias.
Un 11\% describen sus familias como negativas, frustrantes y terribles. Así mismo un 13\% no saben como definir el encuentro relacional con sus familiares.

Sin duda estos resultados pueden ser contrastados con los presentados por González (2006) quien trabajó acerca de la necesidad de apoyo psicológico de los estudiantes hijos de estas familias. Así puede sostenerse como la familia también es susceptible de atención. Además González (2007) ha confirmado como muchas veces esta tensión constituye un problema de salud familiar.

A continuación se muestra una gráfica que resume la alteridad familiar presente en los grupos estudiados.

\section{GRÁFICO 1 ALTERIDAD FAMILIAR}

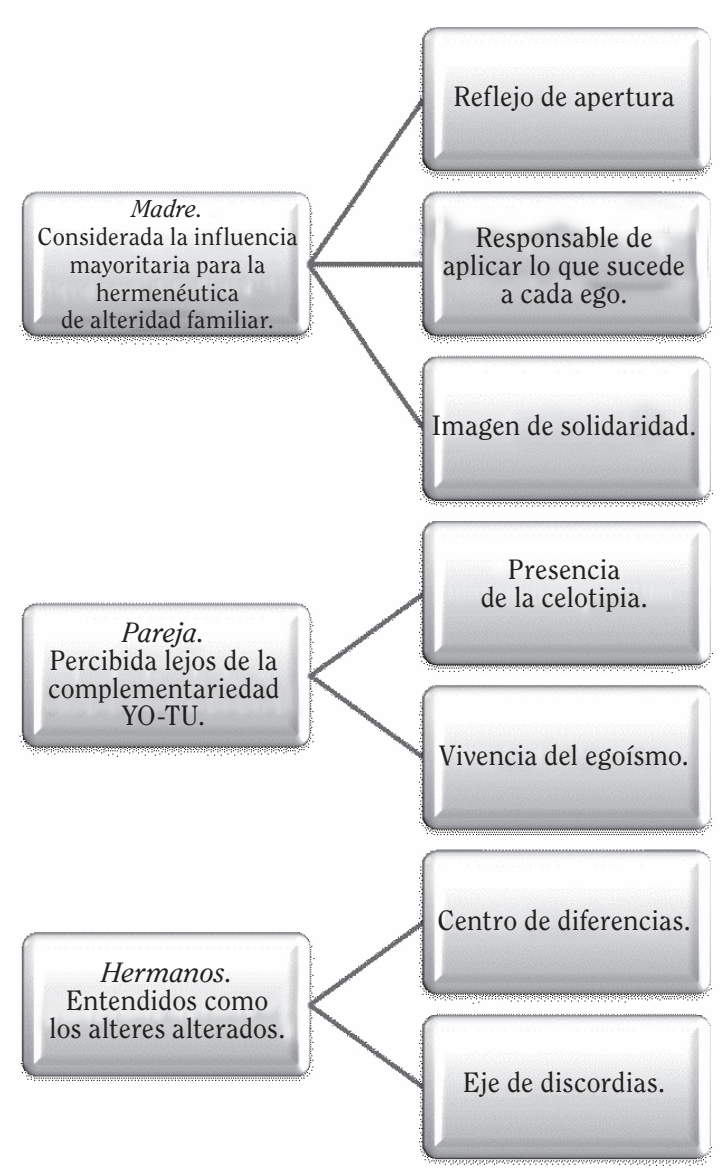


5. ALTERIDAD CON ROSTRO FAMILIAR: ENCUENTRO

Entre las dimensiones positivas más relevantes de las familias abordadas se encuentra el apoyo y la igualdad (87\%). Algunos miembros aceptan su hogar como armonioso (32\%) y agradable (49\%). En estos casos las dificultades son sublimadas y les dan fuerza para seguir adelante (R26).

En general la generosidad también está presente (55\%). Por otro lado existe un encuentro basado en la comunicación (64\%). Así pues resulta fácil comprender como la mayoría de los miembros mantienen una imagen consolidada de la familia (R29) y la mayoría asume que estos aspectos le ayudan a enfrentar las situaciones cotidianas que les toca vivir.

Ahora bien, este encuentro puede entenderse como un medio de supervivencia porque prevalece a pesar de las alteraciones familiares.
Kliksberg (2005) explica que estas familias viven una actividad marcada por la necesidad y la solidaridad fruto de la dependencia del otro. Morandé (2006) expresa igualmente que un elemento importante en la sobrevivencia $y$ confianza de estas familias es la importancia de la reciprocidad.

En este sentido la necesidad de convivir para sobrevivir hace que el encuentro constituya una manera de afrontamiento para la superación de las desavenencias. Desde esta perspectiva cabe considerar a Ruano y Serra (2001) quienes estudiaron que ante los conflictos o situaciones estresantes deviene un cúmulo de interacciones familiares a las cuales llamaron afrontamiento.

El reordenamiento de estas ideas permite afirmar que los núcleos familiares presentan polaridad de acuerdo con la interacción. El siguiente gráfico expresa la dimensión producida por la alteridad vivida en los miembros de las familias.

\section{GRÁFICO 2}

\section{INTERACCIÓN PRODUCIDA POR LA ALTERIDAD FAMILIAR}

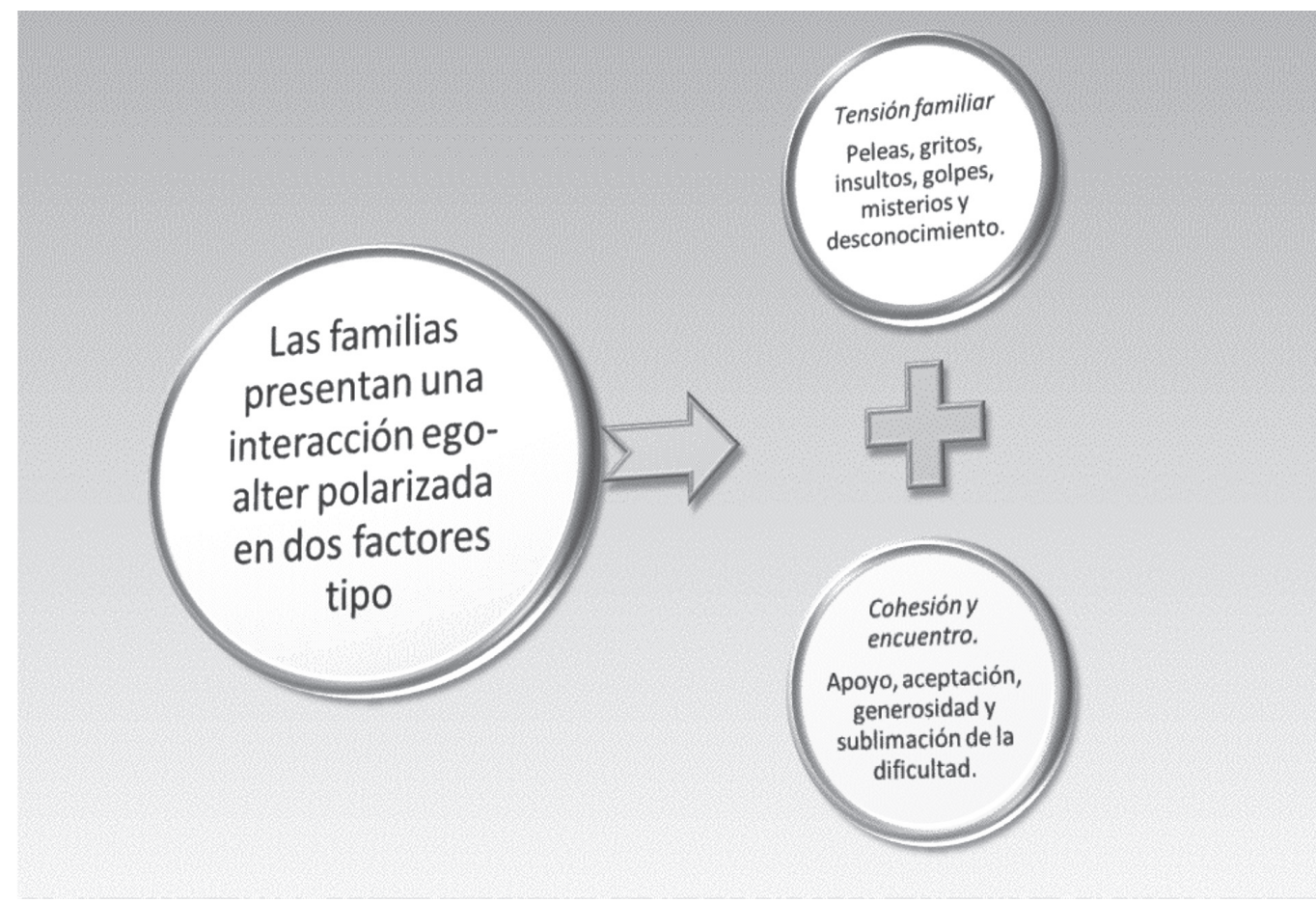




\section{DISCUSIÓN}

Con lo anterior se afirman los resultados de Moreno (2004a) quien halló que la familia venezolana no es convencional, es decir, no está formada por padre, madre e hijos. No hay triángulo sino binomio, pero no en cuanto suma de los miembros, sino en cuanto nudo relacional entre madre e hijos. En efecto, se sostiene sobre sí mismo y se compensa en sí mismo. No necesita nada más para vivir y subsistir en cuanto tal. Esto es una estructura completa y sin fisuras. Una organización binomial, no triangular.

La configuración no está ni en el ser de la madre ni en el ser del hijo ni en la suma de ambos sino en ese vínculo que constituye a la madre en madre $y$ al hijo en hijo $y$ que es uno y el mismo para los dos. Ahora bien, el vínculo emana de la madre y a ella regresa cerrando, así, el nudo. En este sentido y con toda razón, esta familia es matricentrada pues la madre es el centro del vínculo y de los vínculos cuando los binomios se multiplican y se unifican a la vez en ella como vértice de una pirámide.

Dentro de este marco se observa como los resultados se han enfatizado en ubicar las alteraciones principalmente entre los hermanos. En tal sentido pareciese que los padres no percatasen alteraciones entre ellos. Todo ello conlleva a intervenir los núcleos familiares tratando de profundizar en el darse cuenta. Las familias estudiadas parecen obviar que los hijos son consecuencia de la experiencia de alteridad transmitida por ambos padres dentro del núcleo familiar.

En los resultados también se manifiesta, a pesar de la alteridad alterada, creencias que apoyan el encuentro. En tal sentido el hogar es interiorizado por los miembros como positivo $y$ así mantiene una potencialidad para el logro del equilibrio. En efecto el hogar simboliza apoyo e igualdad para los miembros. Así pues esta imagen positiva genera un factor de cohesión que los mantiene unidos.

En otro orden de ideas el análisis precedente permite comprender las diferencias entre las familias pobres estudiadas con cualquiera de los otros lugares o latitudes. En efecto, Degennea y Odile (2005) presentan un panorama muy distinto de las relaciones dadas en las familias del primer mundo $y$ las que tenemos evidentemente en el sector popular venezolano. En el primer caso muchos de los adultos se alejan de sus hogares y mantienen redes relacionales mientras que en las familias investigadas pocos se han alejado de sus hogares $y$ por el contrario viven aun dentro de ellas.

Se plantea entonces la necesidad de abordajes nuevos y contextualizados en los ambientes de pobreza. Evidentemente se trata de familias distintas a las de la mayoría estudiadas en la literatura científica. Así pues, se coincide con Morandé (2006) al señalar que estas familias no solo presentan dificultades materiales, como sus escasos recursos y precariedad de viviendas, sino exclusión social al existir un debilitamiento de los lazos del individuo con la sociedad global, que dificulta el acceso a nuevas oportunidades. Tienen un alto deterioro psicosocial, encontrándose expuestas a situaciones de crisis, al experimentar simultánea y persistentemente obstáculos diversos. Aunado a ello suelen ser familias con miembros relativamente jóvenes $y$ con niños pequeños.

Siendo las cosas así, resulta claro un número de variantes que requieren explicaciones. De hecho un porcentaje importante de las familias estudiadas tienen miembros discapacitados. León et ál. (2003) presenta una vez más como las relaciones interfamiliares son determinantes en el desarrollo del niño de condición especial. Así desde que los padres se enteran de la deficiencia del niño hasta la vejez de esta persona la familia intervendrá ofreciendo herramientas para que se desenvuelvan adecuadamente o no.

Importa $y$ por muchas razones, asumir lo que sucede a nivel familiar para así poder desarrollar políticas acordes con la realidad. Ruano y Serra (2001) recalcan la importancia del apoyo social como estrategia de afrontamiento preferida por familias con educación primaria en lugar de otras estrategias como la reestructuración. La interacción social comunitaria es primordial para que las familias afronten sus problemáticas. 
Por consiguiente con las familias hace falta una intervención más allá de lo convencional. Se ha de lograr una comprensión y una nueva narrativa de los hechos con un estilo psicológico que en términos de Moreno (2004b) se ubicaría en la liberación.

La intervención psicológica va dirigida hacia la generación del autorreconocimiento como familia. Debe señalarse que las familias dentro de su seno han de volcar su mirada hacia la interiorización. En efecto, cada hogar ha de mantenerse alerta en la presencia y significado del padre, la madre, el hermano, el hijo... asumido internamente por cada participante del grupo familiar. Por supuesto que esta experiencia existencial ayudará a no caer en desunión y los alejará de una comprensión del otro basada en la cosificación y el utilitarismo. En definitiva un hogar que vivencia la alteridad camina hacia la empatía, la tolerancia, la reciprocidad, la responsabilidad, el reconocimiento y la complementariedad.

El análisis procedente no permite dejar a un lado el sector escolar. Corbetta, (2006) señala que los docentes se encuentran recep- cionando todos los conflictos sociales en el aula, sin las herramientas necesarias para recibir a ese chico que viene, $y$ resolviendo con estrategias individuales, aquello que la escuela, como institución, aún no pudo hacerle frente. Una concepción de familia y alteridad desde los sectores de pobreza permite una comprensión mejor de los "clientes de esas escuelas". La escuela es para los sectores pobres mucho más que el lugar donde se aprende contenidos, es donde están los amigos, donde se siguen encontrando "profes" que son modelos de vida. La escuela que atiende alumnos de familias pobres invita a ser repensada no sólo como el lugar donde se aprenden contenidos escolares sino como un espacio donde sea posible reflexionar y por sobre todo construir pautas de bienestar para la condición humana siguiendo una educación para la alteridad. Finalmente cabe destacar la necesidad de reflexionar al nivel de la propia familia como microsistema y también por parte de los macrosistemas sociales. A continuación se presenta una gráfica que propicia esta acción.

\section{GRÁFICO 3}

\section{ACCIONES PARA ATENDER LA ALTERIDAD FAMILIAR DE SECTORES COMPROMETIDOS POR LA POBREZA}

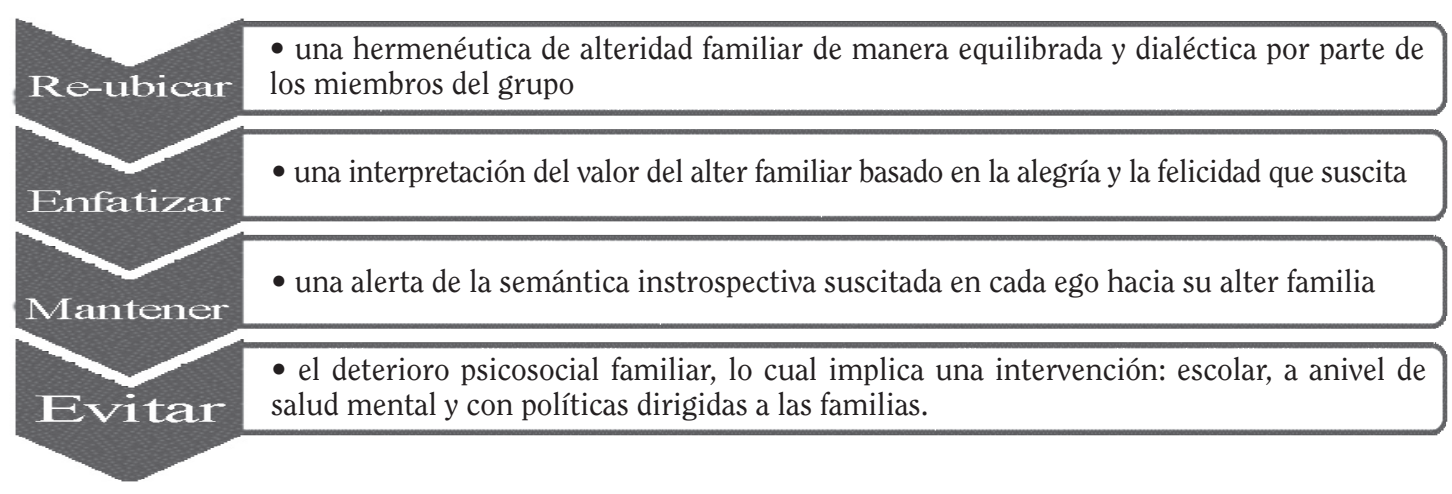

\section{CONCLUSIONES}

Este estudio constituyó la puesta en práctica de la investigación en alteridad dirigida hacia el ámbito familia. Ello permitió realizar una discusión y asumir que la perspectiva de la alteridad es un hecho merecedor de revisión. En efecto, las familias presentaron una tipología de alteridad propia de su contexto y su cultura difícil de percibir con el sentido común. Desde allí se suscitaron muchas líneas para continuar la acción de esta investigación. Sin duda un abordaje social interactivo, una escuela adaptada a la realidad y una intervención psicológica distinta han sido las propuestas más directas. No obstante, con ello es imposible descuidar el 
giro que han de dar las políticas existentes para dirigirse a la familia.

Dentro de este marco la relación de atención e intervención familiar ha de considerar cómo el otro piensa $y$ vivencia a su familiar. En muchos casos al abordar la familia se exploran sus relaciones y sistemas de interacción sin hacer énfasis suficiente en la simbolización que cada uno hace del otro. Se trata pues de ir más allá de las concepciones, pensamientos e ideas de lo que el sujeto familiar reflexiona en torno a su grupo. De esta manera detenerse justo en su alter ego. Resulta claro que la propuesta es hacer hincapié en el significado que el resto de los miembros del grupo familiar tienen de cada ego presente en su sistema. En consecuencia las relaciones con las familias a las que se contacta $y$ con las que se trabaja podrán ir enfatizadas en la alteridad.

\section{REFERENCIAS}

Amara Pace, G. "El adolescente y la familia". Perfiles educativos 60. 1993: 2-10.

Ariza, M. y De Oliveira, O. "Familias en transición y marcos conceptuales en redefinición”. Papeles de población 28. 2001: 9-39.

Arraigada, I. "¿Existen políticas innovadoras hacia las familias latinoamericanas?" Papeles de población 43. 2005: 9-27.

Arruda, A. (Comp.). Representando a alteridade. Petrópolis: Vozes, 1998.

Caride Gómez, J. La alteridad pedagógica, extensión ética de la profesión docente 154. [Revista en línea] 2001: 9-39. En: $<$ http://www.apagina.pt/arquivo/Artigo. asp? $\mathrm{ID}=4443>$ [Consulta: 2006, mayo 29].

Cisterna C. F. "Categorización y triangulación como procesos de validación del conocimiento en investigación cualitativa". Theoria 14 (1). 2005: 61-71.
Colmenares, Yrvis. "La otredad clausurada: prácticas escolares para la mismidad". Heterotopía 27. 2004: 45-59.

Corbetta, S. El rol de la escuela en los sectores pobres. [Documento en línea]. Instituto Internacional de Planeamiento de la Educación, IIPE-UNESCO, 2006. En: <http://www.rieoei.org/deloslectores/ 1520 Corbetta.pdf > [Consulta: 2006, mayo 29].

De Viana, M. "La familia en la clase media". Revista SIC 592. 1997: 61-68.

Degennea, A. y Odile, M. "The dynamics of personal networks at the time of entry into adult life". Social Networks 27. 2005: 337-358.

Galán, E. y Pinzón, M. "Observatorio nacional de infancia, familia y discapacidad". Psicología desde el Caribe 9. 2002: 125150.

González de Rivera Revuelta, J. L. "Empatía y ecpatía”. Psiquis 25 (6). 2004: 243-245.

González, F. "La escuela como un centro para educar sanando o la educación orientada hacia la vida". Revista Boletín de Investigación Educacional 1 (21). 2006: 215-228.

"La alteridad en la atención especial del autismo". Revista Psicología desde el Caribe 15. 2005: 167-181.

. "Salud mental a nivel familiar desde la perspectiva de alteridad". Revista psicología desde el Caribe 20. 2007: 1-27.

Kliksberg, B. "La familia en América Latina, realidades, interrogantes $y$ perspectivas". Convergencia 12 (38). 2005: 13-41.

Krotz, E. "Alteridad y pregunta antropológica". Revista alteridades 4 (8). 1994: 5-11. 
Laursen, B. "Dyadic and group perspectives on close relationships". International Journal of Behavioral Development 29 (2). 2005: 97-100.

León, R.; Menés, M.; Puértolas, N.; Trevijano, I. y Zabalza, S. "El impacto en la familia del deficiente mental". Revista interuniversitaria de formación del profesorado 17 (2). 2003: 195-212.

Levinas, E. El tiempo y el otro. Barcelona: Paidós, 1993.

Miguel, R. y Vargas, R. "Padre ausente y las repercusiones a nivel psicológico en el niño según diversas perspectivas de análisis". [Revista en línea] Apsique, 2001. En: <http://www.udec.cl/ ivalfaro/ apsique/desa/index.php?texto=padre ausente.html> [Consulta: 2006, diciembre 23]

Morandé, Margarita. "La integración de la sociabilidad $y$ asociatividad en el trabajo con familias marginales". Psykhe 15 (1). 2006: 107-117.

Moreno, A. "Familia así, familia asá". Heterotopía 27. 2004a: 9-25.

"Más allá de la intervención". Heterotopía 26. 2004b: 9-26.
Motrico, E.; Fuentes, M. y Bersabé, R. "Discrepancia en la percepción de los conflictos entre padres e hijos/as a lo largo de la adolescencia”. Anales de psicología 17 (1). 2001: 1-13.

Rodríguez, L. "La imagen del otro en relación a la discapacidad. Reflexiones sobre alteridad". Docencia e investigación 3, 2003. [Revista en línea]. En: <http:// www.uclm.es/PROFESORADO/RICARDO/ Docencia_e_Investigacion/3/Bausa. htm> [Consulta: 2005, septiembre 10]

Ruano, R. y Serra, E. "Estrategias de afrontamiento en familias con hijos adolescentes". Anales de psicología 16 (2). 2001: 199-206.

Stein, E. Sobre el problema de la empatía. Madrid: Trotta, 2004.

Theodosíadis, F. Alteridad ¿la (des)construcción del otro? Bogotá: Magisterio, 1996.

Valadez, I.; Amescua, R.; Quintanilla, R. y Gonzalez, N. "Familia e intento suicida en el adolescente de educación media superior". Archivos en medicina familiar 7. 2005: 69-78.

Vila Merino, E. "Pedagogía de la ética: de la responsabilidad a la alteridad". Althenea Digital 6. 2004: 47-55. 